\title{
Characterization of Patterned Porous Low- $k$ Dielectrics: Surface Sealing and Residue Removal by Wet Processing/Cleaning
}

\author{
Q. T. Le ${ }^{\mathrm{a}, \mathrm{z}}$ E. Kesters, ${ }^{\mathrm{a}, *}$ S. Decoster,${ }^{\mathrm{a}}$ B. T. Chan, ${ }^{\mathrm{a}}$ M. P. Nguyen,,${ }^{\mathrm{a}, \mathrm{c}}$ T. Conard, ${ }^{\mathrm{a}}$ \\ A. Vanleenhove, ${ }^{\mathrm{a}}$ F. Holsteyns, ${ }^{\mathrm{a}, *}$ and S. De Gendt ${ }^{\mathrm{a}, \mathrm{b}, *}$
}

${ }^{a}$ Imec, Kapeldreef 75, 3001 Leuven, Belgium

${ }^{b}$ Department of Chemistry, KULeuven, 3001 Leuven, Belgium

\begin{abstract}
The results described in this paper first demonstrate key differences between a plasma-exposed blanket porous dielectric sample and a patterned structure in terms of surface sealing induced by the patterning plasma using ellipsometric porosimetry characterization. While the blanket CVD organosilicate glass (nominal $k$-value $=2.4, \sim 20 \%$ of porosity) surface was sealed by the $\mathrm{C}_{4} \mathrm{~F}_{8} / \mathrm{CF}_{4}$-based plasma, the surface of the analogous patterned structure of $45 \mathrm{~nm} \mathrm{1/2}$ pitch was found to be completely open after the same patterning plasma process. Second, the surface composition of the patterned feature, type of the residues generated during the plasma etch, and the effect of a subsequent wet clean step are presented. The experimental results demonstrate that the use of a relevant patterned test structure and its characterization represent an appropriate approach for the optimization of the etch and cleaning processes. The removal efficiency of various wet clean solutions, including dilute HF, citric acid, tetramethylammonium hydroxide: $\mathrm{H}_{2} \mathrm{O}_{2}: \mathrm{H}_{2} \mathrm{O}$ mixture, and a slightly alkaline formulated mixture, with regard to polymer residues, $\mathrm{CFx}$, and metal-containing residues, TiFx, can be clearly distinguished using the patterned porous low- $k$ stack.

(C) The Author(s) 2016. Published by ECS. This is an open access article distributed under the terms of the Creative Commons Attribution Non-Commercial No Derivatives 4.0 License (CC BY-NC-ND, http://creativecommons.org/licenses/by-nc-nd/4.0/), which permits non-commercial reuse, distribution, and reproduction in any medium, provided the original work is not changed in any way and is properly cited. For permission for commercial reuse, please email: oa@electrochem.org. [DOI: 10.1149/2.0201603jss] All rights reserved.
\end{abstract}

Manuscript submitted December 8, 2015; revised manuscript received January 12, 2016. Published January 22, 2016.

In back-end of line (BEOL) processing of advanced microelectronic CMOS integrated circuits, conductor lines are made using a damascene approach. For this, dielectric layers are locally etched through a patterned photoresist or metal hardmask layer using plasmas and followed by electroplating of $\mathrm{Cu}$ inside the etched patterns. In order to ensure accurate profile control, etching anisotropy, and minimize degradation of the exposed porous dielectric during the plasma etch, polymerizing plasma chemistries are employed to deposit a thin layer of polymer on the exposed surface..$^{1-3}$ A good balance between the polymerization and the dielectric etch rate allows the polymer film to passivate the sidewalls without affecting the vertical etch rate, resulting in a vertical etch profile. The polymer formed during the dry etch process ("post-etch polymer residues" or PER), mostly fluorocarbon polymers for fluorocarbon-containing plasmas, must be removed prior to deposition of subsequent layers (metal) in the etched features to achieve high adhesion and good coverage. Removal of these polymers is usually performed by a combination of a short plasma treatment and wet clean using chemical solutions, or by wet cleans alone. It is known that this type of fluorocarbon polymer is chemically inert to many existing wet clean solutions, including aqueous solutions such as fluoride ion-containing or highly alkaline solutions, and solvent mixtures. ${ }^{4}$ A short plasma treatment carried out prior to the wet clean step enhances the polymer removal efficiency but also has a drawback of irreversibly damaging the porous dielectrics by material densification, carbon depletion, and increase of hydrophilicity. ${ }^{1,2,5,6}$ An alternative approach for improving the residue removal efficiency involves structural modification of the fluorocarbon polymer together with introduction of polar functional groups into the polymer backbone using ultraviolet (UV) irradiation followed by a wet clean step. The UV sources consisted of a narrow band, single-wavelength UV source with $\lambda=254 \mathrm{~nm}^{4,7}$ or a broad band source of $\lambda>200 \mathrm{~nm}^{8}$ or $\lambda>230 \mathrm{~nm} .^{9,10}$ Fluorine-containing polymers deposited on both blanket surface and patterned low- $k$ containing samples were used for the above studies.

For dry etch plasma and cleaning steps, processing and characterization of blanket, large structures reveals helpful information such as etching kinetics, etch selectivity, change in properties and structure

\footnotetext{
*Electrochemical Society Member

${ }^{2}$ Present Address: Advanced Institute for Materials Research, Tohoku University, Japan.

${ }^{\text {z} E-m a i l: ~ Q u o c T o a n . L e @ i m e c . b e ~}$
}

of the material involved, and in particular for porous dielectrics the modification in porosity, $k$-value, and effect on surface sealing. This screening of blanket materials usually represents a first step in the development processes. However, optimization of the process requires further characterization and understanding of material modification in relevant patterned structures. ${ }^{9-12}$ The first objective of the study reported herein is to investigate the difference between a blanket dielectric sample and a patterned structure, $45 \mathrm{~nm} 1 / 2$ pitch, in terms of surface sealing induced by the patterning plasma using ellipsometric porosimetry (EP) characterization. Another aspect of this study concerns the surface composition of the patterned feature, the type of the residues generated during the plasma etch using $\mathrm{C}_{4} \mathrm{~F}_{8} / \mathrm{CF}_{4}$-based plasma and the effect of a subsequent wet clean step. The efficiency of the removal of two different types of residues by wet chemistries can be clearly demonstrated using the patterned structure of $45 \mathrm{~nm}$ $1 / 2$ pitch.

\section{Experimental}

The porous low- $k$ material used in this study was a CVD organosilicate glass (OSG) material with a target $k$-value of 2.4 , a porosity and mean pore diameter of $\sim 20 \%$ and $1.2 \mathrm{~nm}$, respectively. For blanket OSG film, the film thickness was targeted at about $80 \mathrm{~nm}$. The plasma treatment using a $\mathrm{C}_{4} \mathrm{~F}_{8} / \mathrm{CF}_{4}$-based mixture was performed for $10 \mathrm{~s}$ at room temperature. This exposure time is significantly shorter than what usually applied for a patterned structure in order to preserve a certain film thickness for further characterization, but it is still long enough to have relevant effects to the film. A thickness reduction of $\sim 20 \mathrm{~nm}$ was measured for the low- $k$ film after the etch plasma process. As shown in Figure 1, the $45 \mathrm{~nm} \mathrm{1/2}$ pitch test structure, from the bottom, the stack consists of Si substrate/OSG $(80 \mathrm{~nm}) /$ oxide HM $(30 \mathrm{~nm}) / \mathrm{TiN}(25 \mathrm{~nm}) / \mathrm{BARC} / 193-\mathrm{nm}$ photoresist (PR) layer. The TiN layer was first etched using a $\mathrm{HBr} / \mathrm{Cl}_{2}$ plasma, followed by a BARC and PR strip. The oxide HM and OSG etch was then performed using the same type of plasma as the one used for the blanket OSG film $\left(\mathrm{C}_{4} \mathrm{~F}_{8} / \mathrm{CF}_{4}\right.$-based plasma) in a dielectric etch chamber. All dies are fully covered with the same type of $45 \mathrm{~nm} 1 / 2$ pitch structure.

Two methods, which are commonly used for blanket layer characterization were applied for characterization of the patterned structure used in this study, including EP and X-ray photoelectron spectroscopy (XPS). The refractive index and thickness of the blanket dielectric films were measured using the EP-10 equipped with a 


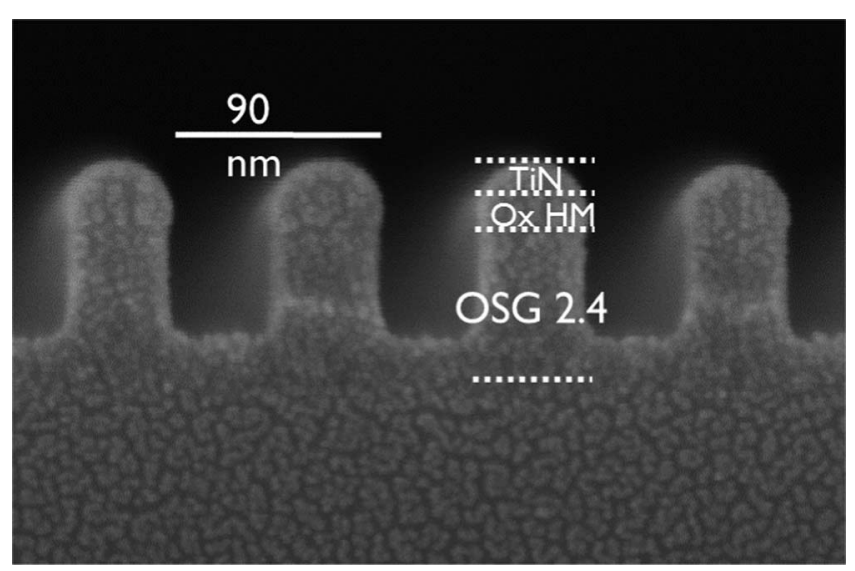

Figure 1. Cross-section SEM image of the 45-nm 1/2 pitch test structure.

multi-wavelength SENTECH 801 ellipsometer at $70^{\circ}$ incidence angle. The same system was also used for ellipsometric porosimetry (EP) measurements of both the blanket film and patterned samples. The ellipsometer is mounted in a vacuum chamber that can be filled with solvent vapor in a controlled way, which can be used for determination of porosity and pore structure. ${ }^{13,14}$ Toluene was used as the adsorbate. The ellipsometry chamber is typically operated at a pressure ranging from $10^{-4}$ Torr to the saturation vapor pressure of the adsorbate. A similar procedure was also applied for the evaluation of the extent of sealing. As described in, ${ }^{15,16}$ the change in ellipsometric angles Delta and Psi during the adsorption are measured by ellipsometry as a function of the adsorbate pressure. In the present study, the sealing assessment is based on the principle that if the surface is sealed, or partially sealed, due to the presence of a dense layer, Delta and Psi will remain unchanged or only show a limited change during the exposure to toluene vapor. On the contrary, if the surface is porous, the solvent adsorbs into the porous network of the dielectric stack leading to significant changes in Delta and Psi.

XPS measurements were carried out using a Thermo VG Scientific Theta 300 system equipped with an $\mathrm{Al} \mathrm{K} \alpha$ source $(1486.6 \mathrm{eV})$ and simultaneous detection of photoelectrons at take-off angles between
22 and 78 degrees. Regarding the analysis mode, all the samples were analyzed following two directions: (a) low- $k$ lines are parallel to the beam toward the XPS analyzer, and (b) low- $k$ lines are perpendicular to the beam. However, as both directions lead to very similar results, the results shown in the next section only concern the perpendicular direction.

All the cleaning experiments were conducted in a beaker set-up under constant agitation using a magnetic bar. HF 0.2 vol.\% was used at room temperature. The cleaning experiments using citric acid 0.2 wt. $\%$, tetramethylammonium hydroxide (TMAH): $\mathrm{H}_{2} \mathrm{O}_{2}: \mathrm{H}_{2} \mathrm{O}$ mixture (the concentrations of TMAH and $\mathrm{H}_{2} \mathrm{O}_{2}$ in the mixture are 1 and 5 vol.\%, respectively), and the formulated mixture (an aqueous mixture with $\mathrm{pH} \sim 7-8$ ) were used at $50^{\circ} \mathrm{C}$. The post-clean samples were then rinsed with DI water ( $2 \mathrm{~min}$ ) and dried with a nitrogen gun (1 $\mathrm{min})$.

\section{Results and Discussion}

Starting from a hydrophobic surface for as-deposited OSG 2.4 (water contact angle $=90-95^{\circ}$ ), the dielectric surface becomes significantly more hydrophilic after being subjected to the $\mathrm{C}_{4} \mathrm{~F}_{8} / \mathrm{CF}_{4}$-based plasma treatment, where the water contact angle is $\sim 35-40^{\circ}$. The change of the hydrophobicity of the surface can be explained by the presence of fluorine-containing species together with an increase in oxygen concentration as indicated by XPS analysis (data not shown). Figure 2a shows the change of Psi as a function of the relative toluene pressure recorded during the toluene adsorption and desorption for the plasma treated blanket OSG film. The change recorded for increasing toluene pressure remained small, corresponding to a slow toluene adsorption, within a small range. Indeed, during the adsorption process, the increase in Psi was less than 0.5 degrees at the relative pressure of 0.5 . The sealing behavior of the plasma-treated film could be caused by the presence of a dense layer at the surface, most probably a silicalike layer induced by the plasma. This silica-like layer plays the role of a barrier to block toluene from penetrating into the porous film. The sealing provided by this modified surface layer is only partial as further increase in ellipsometric angles, Psi (and also Delta - not shown here) was recorded until the complete saturation $\left(\mathrm{P} / \mathrm{P}_{0}=1\right)$. A typical characteristic of a partially sealed surface is the irreversibility of the adsorption-desorption curves as shown in Figure 2a. In addition, after the completion of the desorption process, the Psi value did not return

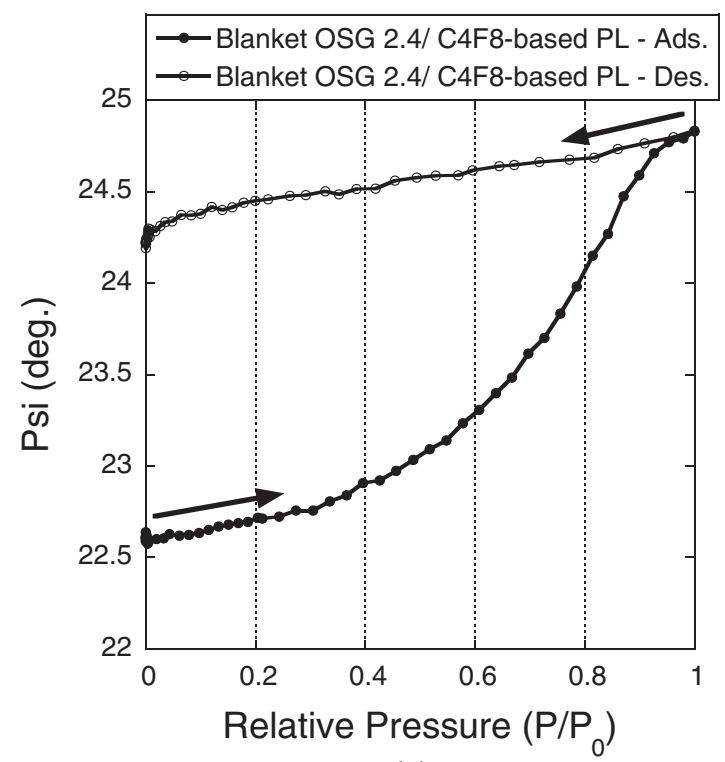

(a)

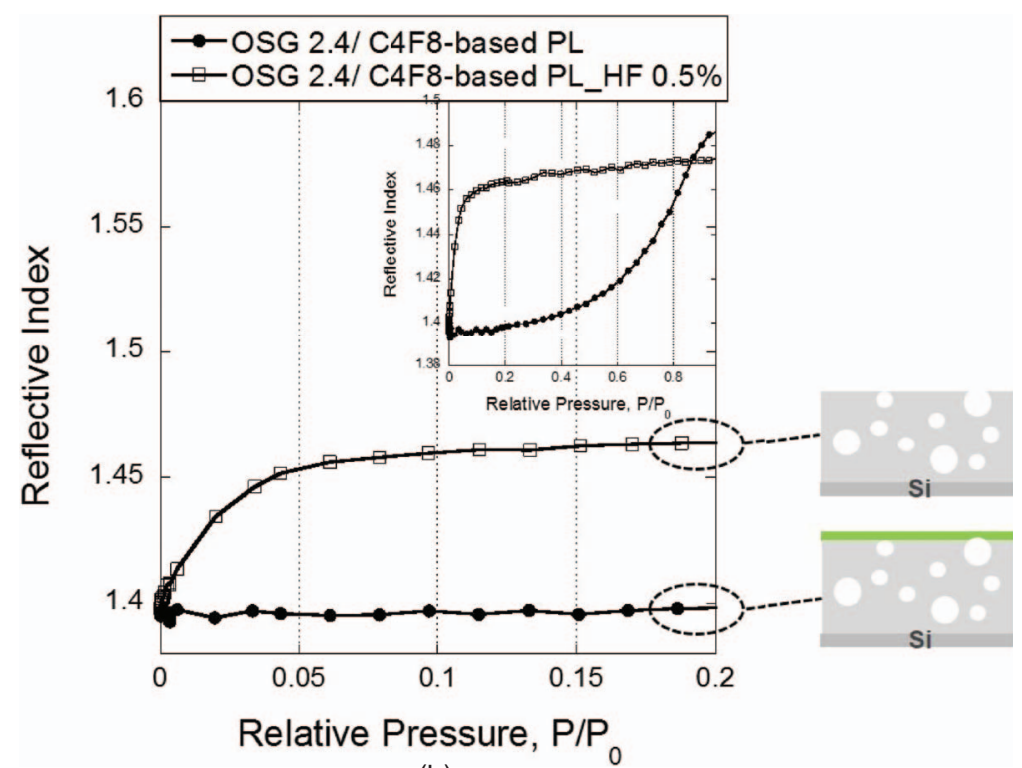

(b)

Figure 2. (a) Evolution of Psi as a function of relative toluene pressure measured during the adsorption and desorption for blanket plasma-treated OSG 2.4 and (b) change in refractive index recorded during the toluene adsorption for blanket plasma-treated OSG 2.4 after immersion in HF $0.5 \%$ solution, as measured by ellipsometric porosimetry. 
to the original value but still remained close to the value measured at $\mathrm{P} / \mathrm{P}_{0}=1$. This can be explained by the fact that the amount of toluene adsorbed into the porous network was not completely pumped out during the desorption phase due to the interconnected porous network, a partial sealing of pore neck together with the presence of the surface sealing layer.

In order to determine the thickness of the sealing layer, several plasma-treated blanket samples were immersed in $0.5 \%$ HF solution at room temperature for different times (10-120 s) aiming to remove the plasma-induced layer from the sample surface. Figure $2 b$ shows the variation of the refractive index as a function of the relative toluene pressure recorded during the adsorption process (pressure increased from 0 to saturation), for the HF-treated sample. As shown in Figure $2 \mathrm{~b}$, unlike the plasma-treated film where a very limited amount of toluene was adsorbed into the film, a significant change in polarization state, and as a consequence the reflective index, was measured indicating the surface is unsealed after removing a layer of $2 \mathrm{~nm}$. The change in RI is caused by the condensation in the pores by toluene (as a measure for porosity change). This behavior is very similar to what was observed for a microporous silica-based low- $k$ film, $k \sim 3$, with a nominal porosity of $\sim 7 \% .^{15}$ This result gives an evidence that for the blanket film the sealing layer only concerns the surface. Starting from a (almost) sealed surface for the plasma-treated porous OSG film, the porous network of the dielectric film bulk became accessible to the solvent vapor once a thin layer from the surface is removed. The plasma-treated OSG 2.4 used in this work was found to be "uncapped" after HF treatment and a thickness reduction of about $2 \mathrm{~nm}$. This strongly suggests that under the plasma conditions described in the experimental part, the thickness of the sealing layer on blanket films is equal or below $2 \mathrm{~nm}$. After cap removal, the open porosity measured at toluene saturation pressure substantially changed from $\sim 3 \%$ (after plasma treatment) to $20 \%$.

In contrast to the plasma-treated blanket film, the adsorption behavior of the $45 \mathrm{~nm} \mathrm{1/2}$ pitch patterned structure is completely different. The rapid change of the polarization state (Psi angle) recorded as soon as the toluene was introduced into the ellipsometry chamber reflects the prompt adsorption of toluene into the porous network (Figure 3 ). As a consequence, this clearly indicates that the patterned low- $k$ surface remains open (unsealed) after the $\mathrm{C}_{4} \mathrm{~F}_{8} / \mathrm{CF}_{4}$-based etch plasma process. The change in Psi measured after a full adsorption was found

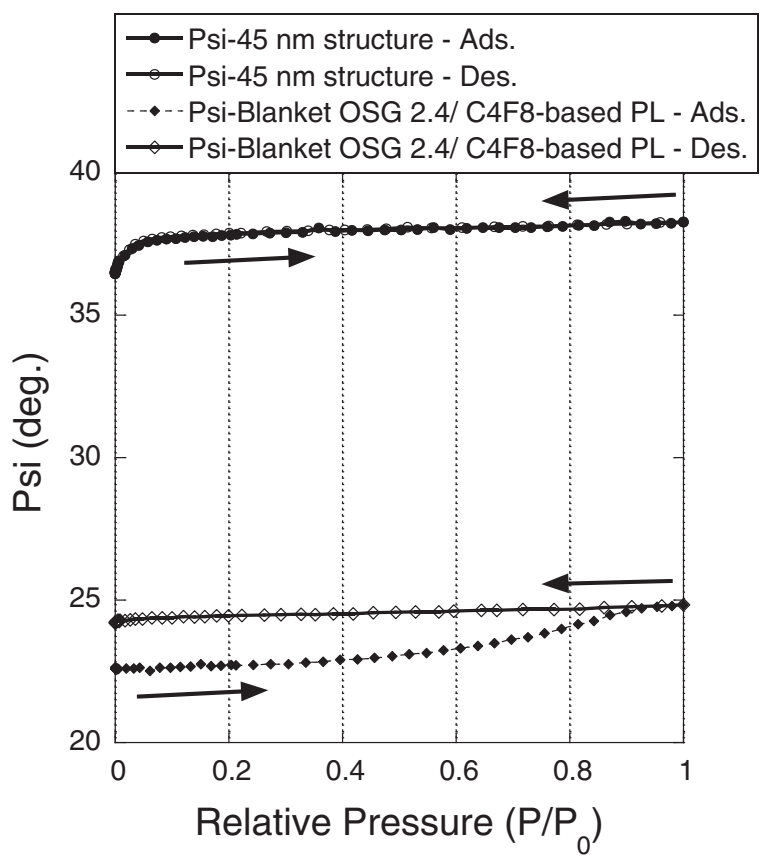

Figure 3. Psi change recorded during adsorption and desorption: comparison between blanket OSG 2.4 and patterned $45 \mathrm{~nm} \mathrm{1/2}$ pitch structure. to be completely reversible, which gives an evidence of a complete interconnected and open porous network. Besides the presence of the TiN and oxide hardmask layers in the stack, the main difference between the patterned structure with respect to the blanket sample is the presence of the vertical surface sidewalls. The horizontal surface of the patterned film interacts in comparable mode as the blanket film with the reactive species such as ions, electrons, photons, produced by the plasma, together with a large dose of ion bombardment. On the contrary, the sidewalls of the patterned sample "only" react with the reactive species, with a minimal dose of ion bombardment. Part of the ions that reached the bottom are believed to bounce off of the pattern and strike the sidewalls with much less energy. It is therefore most likely that ion bombardment is the main factor contributing to the sealing of the blanket sample.

This result also indirectly indicates that the polymer residue present, if there is any, on the sidewall is not thick enough or not uniform to efficiently seal the low- $k$ surface.

Additional experiments were performed to demonstrate the nonsealing character of the patterned surface. To this end, a $45 \mathrm{~nm} \mathrm{1/2}$ pitch sample was first immersed at room temperature in an aqueous solution containing 1\% of surfactant (polyoxyethylene octyl phenyl ether), used as a probe. Figure 4a shows differential IR spectra of the $45 \mathrm{~nm}$ sample stack recorded after immersion in polyoxyethylene octyl phenyl ether solution for 1 and $2 \mathrm{~min}$. These two spectra were obtained by subtraction from the after-etch sample. As a result, the positive absorption bands represent chemical bonds formed or added to the film stack during the immersion. Clearly $\mathrm{C}-\mathrm{H}$ bonds from the probe molecules were detected in the dielectric film after immersion in the solution for $1 \mathrm{~min}$. The spectrum did not change significantly after a longer immersion time indicating that the incorporation already reached its saturation after about $1 \mathrm{~min}$ of immersion. The rather poor resolution and low intensity of the spectra can be explained by the patterned nature of the sample together with the presence of the TiN on the top surface, which plays the role of a "screening" layer.

Figure $4 \mathrm{~b}$ shows the change of Psi as a function of toluene relative pressure recorded for the sample after surfactant incorporation. As a consequence of the surfactant incorporation, the change of Psi was found to decrease substantially. Comparing the magnitude of change of Psi after a complete adsorption suggests that about $65 \%$ of pores were occupied by the surfactant molecules. In addition, as shown in Figure $4 b$, careful monitoring the change of the inflection point as a function of the relative pressure indicates that the mean pore diameter were also substantially decreased. Assuming the initial mean pore diameter of $\sim 1.2 \mathrm{~nm}$ for this OSG 2.4 film, the mean pore diameter of the OSG film after surfactant incorporation is $\sim 0.6 \mathrm{~nm}$. This observation can be explained as follows. During the solution immersion, surfactant molecules diffuse into the bulk via the open pores at the surface and occupy the mesopore volume, which resulted in a lower porosity film. Other pores where the diameters are smaller than the one of the probe molecule remain unfilled, which in turn explains why the film is still porous, despite the substantial decrease of the porosity.

Another aspect of this study concerns the surface composition of the patterned feature characterized using XPS. After the plasma patterning, several peaks corresponding to Si2p, C1s, N1s, O1s, Ti2p, and F1s were detected in a general scan spectrum (not shown). The same elements were detected regardless of the orientation of the lines vs. the XPS analyzer, i.e. parallel or perpendicular. The following spectra were obtained using perpendicular orientation. Considering the collection angle of the XPS system described in the Experimental section and the geometry of the sample based on the cross-section SEM shown in Figure 1, this orientation allows the collection of the composition of the TiN surface, trench bottom, and also the sidewall. One is specifically interested in the type of residues generated during the plasma etch using $\mathrm{C}_{4} \mathrm{~F}_{8} / \mathrm{CF}_{4}$-based plasma and the effect of various wet cleans on the removal of the post-etch residues.

As the constituent layers of the initial stack do not contain fluorine, the high concentration of the F1s peak ( 20 at.\%) recorded after the plasma patterning indicates that substantial amount of fluorinated 


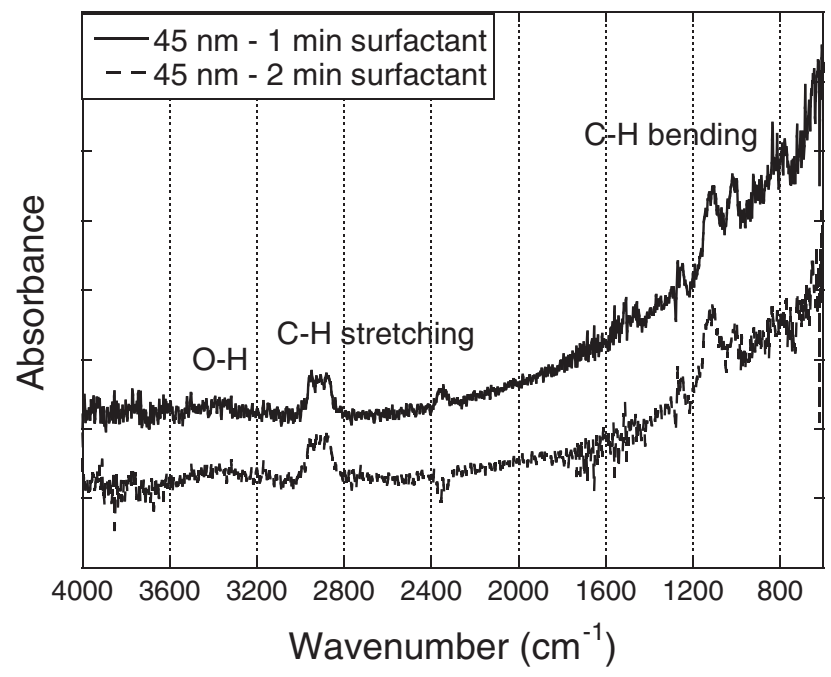

(a)

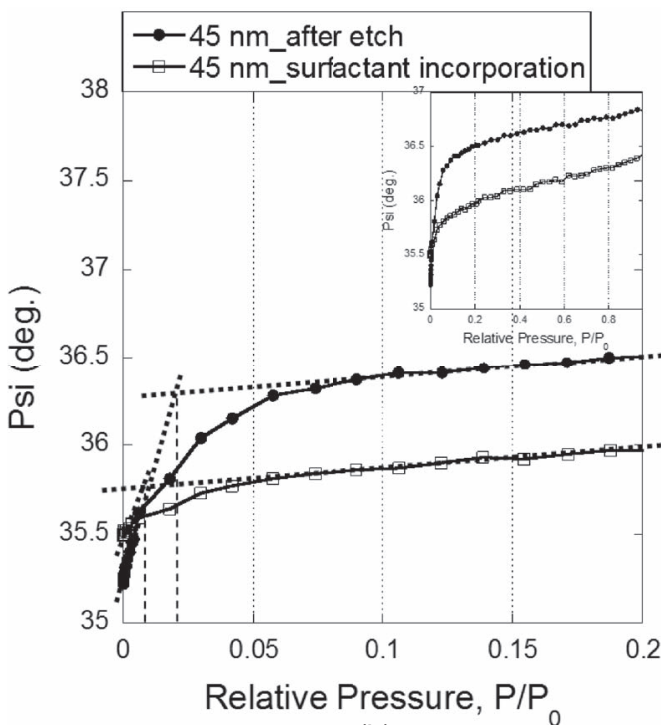

(b)

Figure 4. (a) Differential FTIR spectra recorded after immersion the patterned sample ( $45 \mathrm{~nm} 1 / 2$ pitch structure) in aqueous solution containing $1 \%$ of surfactant, showing the presence of organic molecules in the low- $k$ dielectric layer and (b) Change of Psi measured as a function of relative toluene pressure (adsorption) for the as-patterned $45 \mathrm{~nm} \mathrm{1/2}$ pitch structure and after subsequent incorporation of surfactant molecules.

etch residues are present on both the TiN surface and the dielectric sidewall and bottom. As shown in Figure 5a, the XPS F1s core-level spectrum for the surface after the plasma etch consisted of two main components: the peak at $688.4 \mathrm{eV}$ can be assigned to fluorine bonded to carbon, C-F bonds. ${ }^{17,18}$ The peak centered at $\sim 684.6 \mathrm{eV}$ corresponds to fluorine bonded to titanium, F-Ti bonds, ${ }^{11,17}$ which suggests that the fluorine-containing residues are not only of CFx-type polymer but also TiFx.

Figure $5 b$ shows the Ti2p spectrum of the surface after the plasma etch, the low binding energy peak at $459.1 \mathrm{eV}$ can be assigned to the $\mathrm{Ti} 2 \mathrm{p}_{3 / 2}$ component, while the high binding energy peak at $464.8 \mathrm{eV}$ is primarily due to the Ti $2 \mathrm{p}_{1 / 2}$ component. The binding energy of these peaks indicates that the TiN layer initially deposited on the stack is mostly $\mathrm{TiO}_{2}$ at the surface. The thickness of the $\mathrm{TiO}_{2}$ surface layer is estimated to be thinner than $5 \mathrm{~nm}$, as an additional signal from TiN is also detected at $\sim 455.2 \mathrm{eV} .{ }^{17}$ Note that Ti-F bond is not clearly identified in the spectrum as the TiFx is expected to appear at $\sim 461-$ $463.5 \mathrm{eV}\left(\mathrm{Ti} 2 \mathrm{p}_{3 / 2}\right) .{ }^{17}$ It is difficult to completely resolve the chemical shifts in the Ti2p spectrum due to the overlap of several types of Ti compounds including $\mathrm{TiO}_{2}$, TiN, and TiFx..$^{17,19,20}$ The presence of the latter is however strongly supported by the observed F1s spectrum as discussed above.

The efficiency of the removal of CFx and TiFx by wet chemistries can be clearly distinguished using this type of test structure. The

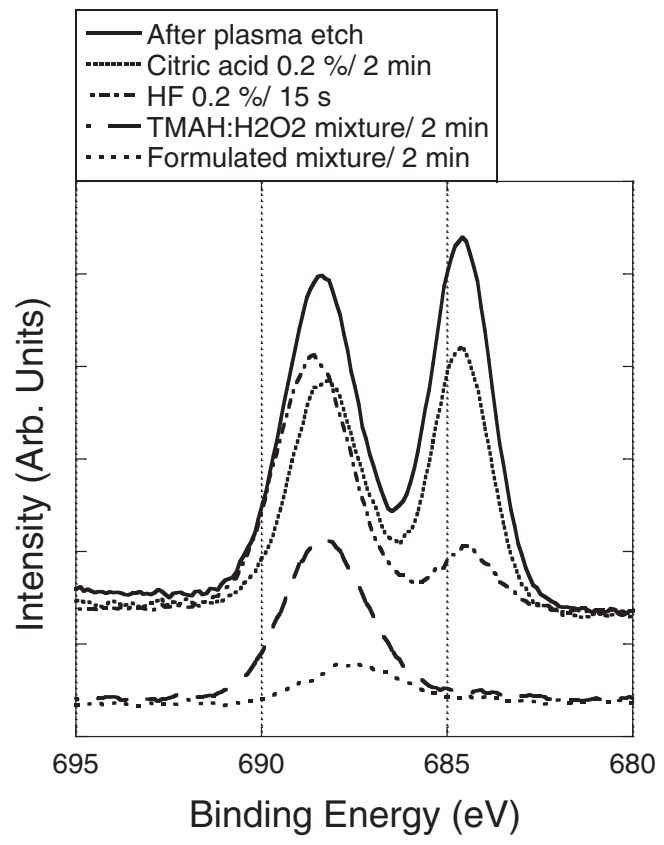

(a)

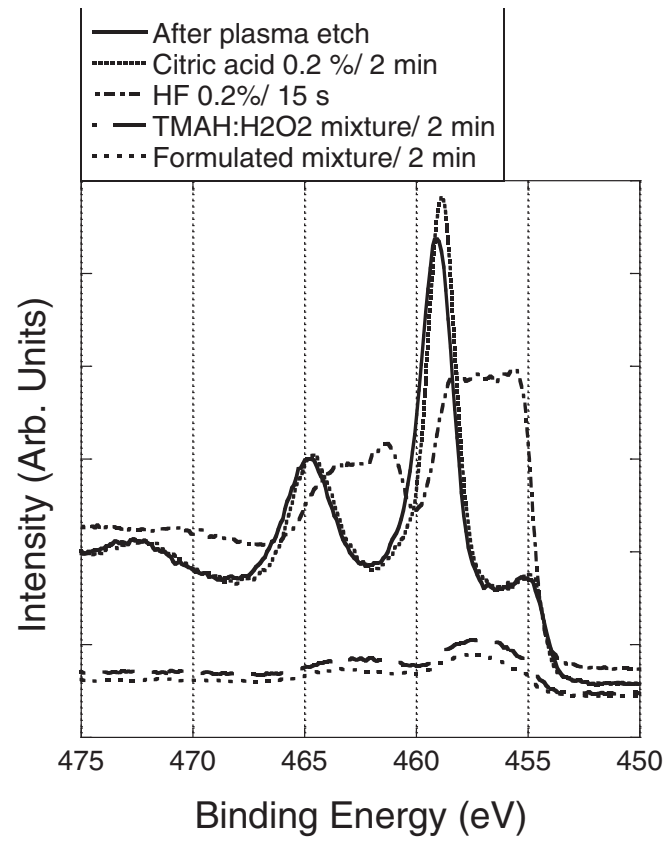

(b)

Figure 5. (a) F1s XPS spectra and (b) Ti2p spectra recorded for the patterned $45 \mathrm{~nm} 1 / 2$ pitch structure after cleaning using various chemistries. 
F1s and Ti2p core-level spectra recorded after various wet cleans are shown in the same figures as above (Figures 5a and 5b). Clearly, a clean using citric acid $0.2 \%$ for 2 min only resulted in little removal of CFx and TiFx, while dilute HF and the TMAH: $\mathrm{H}_{2} \mathrm{O}_{2}: \mathrm{H}_{2} \mathrm{O}$ mixture are efficient for TiFx removal but have only very limited removal of CFx polymer. On the contrary, the formulated mixture is efficient for the dissolution of both $\mathrm{CFx}$ and $\mathrm{TiFx}$ residues, where the F-C component in the F1s spectrum was substantially decreased in intensity (remaining fluorine $\sim 2$ at. $\%$ ) and the Ti-F peak completely disappeared. The clean using the formulated mixture shown here is for comparison purpose, and also in order to demonstrate that the removal of CFx polymer generated during the plasma is possible in one step with optimized process. These results also indirectly suggest that in order to enhance the efficiency of HF-based or TMAH-based cleaning solution, a reactive component aiming at $\mathrm{CFx}$ dissolution should be added to the solutions. The Ti2p spectra presented in Figure $5 \mathrm{~b}$ give additional information on the type of the materials removed during the wet clean process. For instance, citric acid clean has almost no impact on the dissolution of $\mathrm{TiO}_{2}$ and TiN layers. HF $0.2 \%$ partially dissolved the $\mathrm{TiO}_{2}$ layer at the surface thus started to uncover the TiN layer underneath. And finally, both the TMAH: $\mathrm{H}_{2} \mathrm{O}_{2}: \mathrm{H}_{2} \mathrm{O}$ and formulated mixtures were able to dissolve TiN. The TiN layer was almost completely removed after immersion in the mixtures for $2 \mathrm{~min}$. The TiFx residues, most likely deposited mainly on the TiN surface, were hence removed together with the TiN.

\section{Summary}

The outcome of the work described in this paper is not only of interest for the optimization of the etch and cleaning processes in BEOL, but also demonstrate that the use of a relevant patterned test structure and its characterization represent an appropriate approach for the optimization of these processes. With regard to surface sealing, experimental results showed the difference between a blanket porous dielectric sample and a patterned structure after etching using the same dry etch process, $\mathrm{C}_{4} \mathrm{~F}_{8} / \mathrm{CF}_{4}$-based plasma. While the porous low- $k$ surface was sealed by the plasma, the surface of the patterned structure of $45 \mathrm{~nm} \mathrm{1/2}$ pitch was found to be completely open after the same patterning plasma process. In addition, the results showed that substantial amount of fluorinated etch residues were detected on both the TiN surface and the dielectric trench sidewall and bottom. In terms of removal of post-etch residues, the efficiency of various wet clean solutions can be clearly distinguished for polymer residues, $\mathrm{CFx}$, and metal-containing residues, $\mathrm{TiFx}$, using the same patterned porous low$k$ stack. For instance, both dilute $\mathrm{HF}$ and TMAH: $\mathrm{H}_{2} \mathrm{O}_{2}: \mathrm{H}_{2} \mathrm{O}$ mixture are efficient for TiFx removal but only showed incomplete dissolution of CFx polymer. Optimized chemical solutions or mixtures to be selected for wet clean process should remove both the polymer CFx and Ti-containing residues, without modifying the properties of the dielectric material in the stack.

\section{Acknowledgments}

The authors thank D. Parnell (TEL) for her support and fruitful discussions on plasma treatment experiments.

\section{References}

1. H. Shi et al., "Plasma Processing of Low- $k$ Dielectrics", in Advanced Interconnects for ULSI Technology, Ed. M. R. Baklanov, P. S. Ho, and E. Zschech, John Wiley \& Sons Ltd. (2012) Doi: 10.1002/9781119963677.

2. M. R. Baklanov, J.-F. de Marneffe, D. Shamiryan, A. M. Urbanowicz, H. Shi, T. V. Rakhimova, H. Huang, and P. S. Ho, J. Appl. Phys., 113, 041101 (2013).

3. M. Darnon, T. Chevolleau, D. Eon, R. Bouyssou, B. Pelissier, L. Vallier, O. Joubert, N. Posseme, T. David, F. Bailly, and J. Torres, Microelectron. Eng., 85, 2226 (2008).

4. Q. T. Le, J-F. de Marneffe, T. Conard, I. Vaesen, H. Struyf, and G. Vereecke, J. Electrochem. Soc., 159, H208 (2012).

5. N. Posseme, T. Chevolleau, O. Joubert, V. Vallier, and N. Rochat, J. Vac. Sci. Technol. $B, 22,2772$ (2004).

6. F. Bailly, T. David, T. Chevolleau, M. Darnon, N. Posseme, R. Bouyssou, J. Ducote, O. Joubert, and C. Cardinaud, J. Appl. Phys., 108, 014906 (2010).

7. Q. T. Le, S. Naumov, T. Conard, A. Franquet, M. Müller, B. Beckhoff, C. Adelmann, H. Struyf, S. De Gendt, and M. R. Baklanov, ECS J. Solid State Sci. Technol., 2, N93 (2013).

8. Q. T. Le, I. Simms, H. Yue, I. Brown, E. Kesters, G. Vereecke, H. Struyf, and S. De Gendt, Microelectron. Eng., 114, 136 (2014).112.

9. T. Mukherjee, S. Rimal, S. Koskey, O. Chyan, K. J. Singh, and A. M. Myers, ECS Solid State Lett., 2, N11 (2013).

10. T. Mukherjee, S. A. Berhe, A. Goswami, O. Chyan, K. J. Singh, and I. Brown, Appl. Mater. Interfaces, 7 (9), 5051 (2015).

11. M. Darnon, T. Chevolleau, D. Eon, L. Vallier, J. Torres, and O. Joubert, J. Vac. Sci. Technol. B, 24, 2262 (2006).

12. R. Bouyssou, M. El Kodadi, C. Licitra, T. Chevolleau, M. Besacier, N. Posseme, O. Joubert, and P. Schiavone, J. Vac. Sci. Technol. B, 28, L31 (2010).

13. M. R. Baklanov, K. P. Mogilnikov, V. G. Polovinkin, and F. N. Dultsev, J. Vac. Sci. Technol. B, 18, 1385 (2000).

14. M. R. Baklanov and K. P. Mogilnikov, Microelectron. Eng., 64, 335 (2002).

15. Q. T. Le, M. R. Baklanov, E. Kesters, A. Azioune, H. Struyf, W. Boullart, J.-J. Pireaux, and S. Vanhaelemeersch, Electrochem. Solid-St. Lett., 8 (7), F21 (2005).

16. F. Iacopi, C. Zistl, C. Jehoul, Z. Tokei, Q. T. Le, A. Das, C. Sullivan, G. Prokopowicz, D. Gronbeck, M. Gallagher, J. Calvert, and K. Maex, Microelectron. Eng., 64, 351 (2002).

17. P. J. Matsuo, T. E. F. M. Standaert, S. D. Allen, G. S. Oehrlein, and T. J. Dalton, J. Vac. Sci. Technol. B, 17, 1435 (1999).

18. R. d'Agostino, F. Fracassi, and C. Pacifico, J. Appl. Phys., 72, 4351 (1992).

19. T. Sultana, G. L. Georgiev, G. Auner, G. Newaz, H. J. Herfurth, and R. Patwa, Appl. Surf. Sci., 255, 2569 (2008).

20. S. Hashimoto, A. Murata, T. Sakurada, and A. Tanaka. J. Surf. Ana. 10, 12 (2003) 